others were lymphadenopathy, orbital pseudotumor, pancreas and salivary glands in decreasing order (Table). Twenty-four (46.1\%) of patients had localized involvement. Corticosteroids were mainstay of treatment in $92.5 \%$ of patients, and in $57.5 \%$ with any immunosuppressive agents as first line treatment. Rituximab has been used for cases resistant to previous treatment or with relapses in $19(47.5 \%)$ of patients. A complete response was achieved in $52.5 \%$ of patients and partial response ( $<50 \%$ of regression) in $40 \%$. Two patients deceased due to IgG4-RD attributed problems and no malignancy was observed (median follow up: 18 months). Conclusions: We observed similar features with previous European cohorts however no male predominance was seen. Even though conventional immunosuppressives were used in more than half of patients, treatment had switched to rituximab $\sim 50 \%$ patients owing to resistance or relapses.

Disclosure of Interest: None declared

DOI: 10.1136/annrheumdis-2017-eular.4315

\section{FRI0613 H-FERRITIN AND PRO-INFLAMMATORY CYTOKINES ARE INCREASED IN THE BONE MARROW OF ADULT PATIENTS AFFECTED BY MACROPHAGE ACTIVATION SYNDROME}

P. Ruscitti ${ }^{1}$, P. Cipriani ${ }^{1}$, F. Ciccia ${ }^{2}$, P. Di Benedetto ${ }^{1}$, A.R. Lizzi $^{3}$, V. Liakouli ${ }^{1}$, O. Berardicurti ${ }^{1}$, F. Carubbi ${ }^{1}$, G. Guggino ${ }^{2}$, G. D'Andrea ${ }^{3}$, G. Triolo ${ }^{2}$, R. Giacomelli ${ }^{1} .{ }^{1}$ Division of Rheumatology, Department of Biotechnological and Applied Clinical Science, School of Medicine, University of L'Aquila, L'Aquila; ${ }^{2}$ Division of Rheumatology, Department of Internal Medicine, University of Palermo, Palermo; ${ }^{3}$ Department of Biotechnological and Applied Clinical Science, School of Medicine, University of L'Aquila, L'Aquila, Italy

Background: During macrophage activation syndrome (MAS), an inflammatory life-threatening syndrome, extremely high levels of serum ferritin may be observed [1]. Ferritin is an intracellular iron storage protein comprising 24 subunits that may be divided in heavy $(H)$ subunits and light $(L)$ subunits, based on their molecular weight [2]. The $\mathrm{H}$-/L-subunits ratio may change, depending on the specific tissue and the physiologic status of the cell. In the normal condition, ferritin enriched in $L$ subunits ( $L$-ferritin) has been found in the liver and in the spleen, whereas the ferritin enriched in $\mathrm{H}$ subunits ( $\mathrm{H}$-ferritin), may be mainly observed in the heart and kidneys [2]

Objectives: We investigated the tissue expression of both $\mathrm{H}$-and $\mathrm{L}$-ferritin as well as the macrophage subsets expressing these molecules, in the inflammatory BM infiltrate of MAS patients. In addition, the co-expression of IL-1 $\beta, T N F$, IFN- $\gamma$ and $\mathrm{H}$ - or $\mathrm{L}$ ferritin, within the inflammatory cells, was assessed. Finally, we explored if the imbalance between $\mathrm{H}$-ferritin and $\mathrm{L}$-ferritin as well as the number of ferritin positive cells may be considered helpful bio-markers to assess the severity of these patients.

Methods: We analysed the bone marrow (BM) biopsies, by immunofluorescence of 10 adult MAS patients affected by rheumatic disease to assess the presence of: i. both $\mathrm{H}$ - and L-ferritin; ii. the number of CD68+/H-ferritin+ and CD68+/L-ferritin+; iii. the tissue pro-inflammatory cytokines, IL-1 $\beta$, TNF, IFN- $\gamma$; and we correlated these data with clinical and laboratory data. Furthermore, the presence of ferritins was assessed in the sera of the same patients by western blot analysis.

Results: We observed an increased tissue expression of $\mathrm{H}$-ferritin and of proinflammatory cytokines (IL-1 $\beta$, TNF, IFN- $\gamma$ ). Western blot analysis, in the sera, of $\mathrm{H}$-ferritin mirrored data on the tissue. Furthermore, an increased number of CD68+/H-ferritin+ cells and an infiltrate of cells co-expressing $\mathrm{H}$-ferritin and IL-12, suggesting an infiltrate of M1 macrophages, were observed.

Tissue $\mathrm{H}$-ferritin levels correlated with the decreased counts of WBC $(p=0.01)$ and PLT $(p=0.0001)$; with the increased values of serum ferritin $(p=0.012)$ and C-reactive protein (CRP) $(p=0.0058)$; and with the tissue expression of $\mathrm{IL}-1 \beta(p=0.006)$. The number of the $\mathrm{CD} 68+/ \mathrm{H}$-ferritin + cells correlated with the decreased counts of WBC $(p=0.03)$ and PLT $(p=0.0007)$, and with the increased serum ferritin levels $(p=0.0088)$ and CRP $(p=0.049)$. The analyses concerning tissues L-ferritin as well as the number of CD68+/L-ferritin+ cells and the same parameters failed to show any significant result.

Conclusions: We observed an increased tissue expression of $\mathrm{H}$-ferritin associated with an increased expression of IL-1 $\beta$. Interestingly, in the BM inflammatory infiltrate an increased number of $\mathrm{CD} 68+/ \mathrm{H}$-ferritin+ cells was shown. Of note, tissue expression of $\mathrm{H}$-ferritin as well as the number of $\mathrm{CD} 68+/ \mathrm{H}$-ferritin+ significantly were associated with the hematological involvement of the disease, suggesting possible bio-markers to assess the severity of these patients. References:

[1] Ramos-Casals M, et al. Lancet. 2014;383:1503-16.

[2] Rosário C, et al. BMC Med. 2013;11:185.

Disclosure of Interest: None declared

DOI: 10.1136/annrheumdis-2017-eular.5420

\section{FRI0614 FREQUENCY OF ORGAN MANIFESTATIONS IN CHRONIC SARCOIDOSIS}

R. Bergner ${ }^{1}$, K. de Groot ${ }^{2}$, G.A. Müller ${ }^{3}$, P. Korsten ${ }^{3} .{ }^{1}$ Medizinische Klinik A, Klinikum Ludwigshafen, Ludwigshafen: ${ }^{2}$ Medizinische Klinik III, Sana Klinikum Offenbach, Offenbach; ${ }^{3}$ Klinik für Nephrologie und Rheumatologie,

Universitätsmedizin Göttingen, Göttingen, Germany

Background: Chronic sarcoidosis is a systemic disease of unknown etiology, characterized by the histological finding of granulomas in involved organ systems. The most often affected organ is the lung with approximately $90-95 \%$. Systematic data of organ manifestations other than the lung are scarce and show a wide range from $1-2 \%$ up to $50 \%$ depending on the series.

Methods: We analyzed data of newly diagnosed chronic sarcoidosis in 3 tertiary hospitals. We analyzed data on organ manifestations (OM), type of OM and laboratory findings. The certainty of $\mathrm{OM}$ was classified as grade 0 (not investigated), grade 1 (no sign of OM), grade 2 (clinical sign of OM), grade 3 (signs of OM in laboratory findings or imaging) and grade 4 (histological proven $\mathrm{OM})$, respectively.

Results: We included 151 patients with biopsy-proven chronic sarcoidosis. Mean age was $50.8 \pm 15$ years with a male predominance $(87$ [57.2\%] vs. 65 [42.8\%] patients).

Except for 3 patients, all demonstrated pulmonary involvement. The predominant type of lung involvement was type I (mediastinal lymph node enlargement) in $54.2 \%$ and type II (mediastinal lymph node enlargement and interstitial involvement) in $27.7 \%$.

$96.5 \%$ of patients were investigated for an affection of the kidneys, $97.3 \%$ for hepatic, $92.7 \%$ for skin involvement, $68.8 \%$ for ocular manifestations, $67.5 \%$ for ear, nose, throat (ENT) manifestations and $92 \%$ for cardiac manifestations, respectively.

Grade 3 (imaging/laboratory) and grade 4 (histology) findings were seen in the kidneys in $7.6 / 22.8 \%$, in the liver in $13.3 / 11.9 \%$, in the heart in $10.6 / 0.7 \%$, in the eyes in 6.6/

Conclusions: $\mathrm{OM}$ in chronic sarcoidosis are more frequent than suggested in the current literature, especially renal and hepatic. About $20 \%$ of patients with chronic sarcoidosis suffered from moderate to severe CKD due to sarcoidosis, which is a major organ complication contributing to overall morbidity.

We recommend a systematic screening for OM in all patients with chronic sarcoidosis as it is performed in other systemic rheumatic disease.

Disclosure of Interest: None declared

DOI: 10.1136/annrheumdis-2017-eular.6716

\section{FRI0615 SARCOIDOSIS AND CANCER: DIFFERENT PATTERNS OF ASSOCIATION IN A MULTICENTER COHORT FROM SOUTHERN EUROPE}

R. Pérez-Alvarez ${ }^{1}$, B. Kostov ${ }^{2}$, A. González García ${ }^{3}$, R. Gómez De La Torre ${ }^{4}$, M. Lopez Dupla ${ }^{5}$, B. De Escalante ${ }^{6}$, A. Alguacil ${ }^{7}$, J. Chara ${ }^{8}$, J. Velilla ${ }^{9}$, J. Rascón ${ }^{10}$, J.S. Garcia Morillo ${ }^{11}$, C. Tolosa ${ }^{12}$, E. Fonseca ${ }^{13}$, M. Bonet ${ }^{14}$ J.L. Callejass ${ }^{15}$, G. de la Red ${ }^{16}$, E. Calvo ${ }^{17}$, A. Gómez Lozano ${ }^{18}$, E. Peral ${ }^{19}$, J.F. Gómez Cerezo ${ }^{20}$, G. Cruz ${ }^{21}$, P. Perez ${ }^{22}$, S. Rodríguez Fernández ${ }^{23}$, B. Pinilla ${ }^{24}$, A. Gato ${ }^{25}$, M. Akasbi ${ }^{26}$, A. Robles ${ }^{27}$, I. Ojeda ${ }^{28}$, M.J. Vives ${ }^{29}$, C. Morcillo ${ }^{30}$, M. Penadés ${ }^{31}$, M. De Vicente ${ }^{32}$, M. Ramos-Casals ${ }^{33}$, L. Pallarés ${ }^{10}$, P. Brito-Zerón ${ }^{30,33}$ on behalf of the SARCOGEAS-SEMI Registry. ${ }^{1}$ Hosp Alvaro Cunqueiro, Vigo; ${ }^{2}$ IDIBAPS, Barcelona; ${ }^{3}$ Hosp Ramón y Cajal, Madrid; ${ }^{4}$ HUCA, Oviedo; ${ }^{5}$ Hosp Joan XXIII, Tarragona; ${ }^{6}$ Hosp Clínico, Zaragoza; ${ }^{7}$ Hosp Virgen de la Salud, Toledo; ${ }^{8}$ Hosp Josep Trueta, Girona; ${ }^{9}$ Hosp Miguel Servet, Zaragoza; ${ }^{10}$ Hosp Son Espases, Palma de Mallorca; ${ }^{11}$ Hosp Virgen del Rocio, Sevilla; ${ }^{12}$ Hosp Parc Taulí, Sabadell; ${ }^{13}$ Hosp Cabueñes, Gijón; ${ }^{14}$ Althaia,

Manresa; ${ }^{15}$ Hosp San Cecilio, Granada; ${ }^{16}$ Hosp Esperit Sant, Santa Coloma;

${ }^{17}$ Hosp San Jorge, Huesca; ${ }^{18}$ Hosp Sta Caterina, Girona; ${ }^{19}$ Hosp Virgen Macarena, Sevilla; ${ }^{20}$ Hosp Infanta Sofía, Madrid; ${ }^{21}$ Hosp de Poniente, Almería;

${ }^{22}$ Hosp Puerta del Mar, Cádiz; ${ }^{23}$ Hosp da Barbanza, A Coruña; ${ }^{24}$ Hosp Gregorio Marañón, Madrid; ${ }^{25} \mathrm{CH}$, Albacete; ${ }^{26}$ Hosp Infanta Leonor; ${ }^{27}$ Hosp la Paz, Madrid; ${ }^{28}$ Hosp Valle del Guadiato, Córdoba; ${ }^{29}$ San Joan de Déu, San Boi;

${ }^{30}$ Hosp CIMA-Sanitas, Barcelona; ${ }^{31}$ Hosp de Manises, Valencia; ${ }^{32}$ Hosp Nuestra Señora del Prado, Talavera; ${ }^{33}$ Hosp Clínic, Barcelona, Spain

Objectives: To evaluate the temporal association between the diagnosis of neoplasia and sarcoidosis in a large cohort of Spanish patients with sarcoidosis. Methods: In January 2016, the Autoimmune Diseases Study Group (GEASSEMI) created a national registry (SARCOGEAS) of patients with sarcoidosis Sarcoidosis was diagnosed with the criteria proposed by the ATS/ERS/WASOG 1999 statement, and extrathoracic disease by the 2014 WASOG instrument. Diagnosis of neoplasia was recorded before and after the diagnosis of sarcoidosis. Results: The cohort included 1082 patients (82\% biopsy-proven, 618 women, mean age 47yrs). Association with neoplasia was detected in 135 (13\%) patients who developed 140 neoplasms ( 110 solid and 30 hematological neoplasia). The neoplasia more frequently reported were breast $(n=18)$, lymphoma $(n=16)$, non-melanoma skin $(n=15)$ and colon $(n=15)$. Association with neoplasia was more frequent in patients born in Spain $(97 \%$ vs $86 \%, p<0.001$, OR 4.06$)$, older patients ( 55 vs $46 y$ rs, $p<0.001$, OR 1.03 ) and those with bone marrow involvement ( $14 \%$ vs $4 \%, p<0.001$, OR 3.64). Patients in whom cancer preceded the diagnosis of sarcoidosis had a higher frequency of sarcoidosis diagnosed incidentally ( $20 \%$ vs $4 \%, p=0.011)$ and a lower frequency of ocular sarcoidosis ( $3 \%$ vs $16 \%, p=0.016)$. Patients with associated hematological neoplasia had a higher frequency of ENT ( $13 \%$ vs $1 \%, p=0.009)$ and bone marrow $(33 \%$ vs $9 \%, p=0.002)$ involvements in comparison with patients with associated solid neoplasia.

Conclusions: Association between sarcoidosis and cancer was found in $13 \%$ of patients ( $80 \%$ solid and $20 \%$ hematologic malignancies). Elderly patients and those born in Spain were at high risk of having associated cancer. Asymptomatic 